\title{
Radiation hard semiconductor devices for very high luminosity colliders
}

\author{
Marta Baselga Bacardit* \\ CNM-IMB, CSIC Barcelona \\ E-mail: marta.baselgaeimb-cnm.csic.es
}

The next upgrades of the HL-LHC (High Luminosity-Large Hadron Collider) are scheduled to reach fluences of $2 \times 10^{16} \mathrm{n}_{\mathrm{eq}} / \mathrm{cm}^{2}$. Silicon detectors will be exposed to high fluences of radiation, and RD50 (Radiation Hard Semiconductor Devices For High Luminosity Colliders) is a CERN R\&D collaboration devoted to develop radiation hard silicon detectors for the HL-LHC. The collaboration explores different research fields structured in different areas: defect and material characterization explores the macroscopic properties of materials before and after irradiation, detector characterization tests the devices with different techniques, new detector structures developes new devices such as Low Gain Avalanche Detectors (LGAD) or 3D detectors and full detector systems integrates the electronic to the detector and studies the performance under high fluences.

24th International Workshop on Vertex Detectors

\section{1-5 June 2015}

Santa Fe, New Mexico, USA

\footnotetext{
${ }^{*}$ Speaker.

${ }^{\dagger}$ On behalf of the RD50 collaboration.
} 


\section{Introduction}

During the next upgrades of the Large Hadron Collider (LHC), detectors from the inner tracker are foreseen to receive fluences up to $2 \times 10^{16}-1 \mathrm{MeV}$ equivalent neutron $\mathrm{cm}^{2}$ (or $2 \times 10^{16} \mathrm{n}_{\mathrm{eq}} / \mathrm{cm}^{2}$ ). After a run of 10 years, the performance of the innermost LHC silicon detectors will have to cope with high fluences, since integrated luminosities of $3000 \mathrm{fb}^{-1}$ are expected.

RD50 is a CERN R\&D collaboration [1] devoted to develop radiation hard semiconductor detectors for HL-LHC (High Luminosity-Large Hadron Collider). 49 institutes and 280 members belong to RD50, distributed worldwide. The collaboration is active since 2001 and concentrates its efforts in four separated areas: Defect and Material Characterization, Detector Characterization, New Structures and Full Detectors Systems. On the following pages the different areas of research will be described.

\section{Defect and Material Characterization: from microscopic to macroscopic scale}

High energy particles impinging a crystaline lattice create microscopic defects that lead to changes in the silicon detector performance in the macroscopic scale. Charged acceptor and donor defects increase the electric field and change the effective doping, for instance E205a(-/0) and $\mathrm{H} 152 \mathrm{~K}$ are defects that increase the charge trapping leading to a decrease of the Charge Collection Efficiency (CCE) [2], while other defects are responsible for the increment of the leakage current. Defects such as H152(-/0), H140(-/0) and H116(-/0) are responsible for causing the long term annealing effects[3]. Techniques such as TSC (Thermally Stimulated Current) or DLTS (Deep Level Transient Spectroscopy) give a better understanding of the microscopic defects. The study of unirradiated and irradiated samples gives information on the radiation induced defects and their electrical properties. Fig. 1 shows TSC measurements after different fluences of electron irradiations.

As seen in fig. 1, the defect concentrations increases with the radiation. The identification and a consistent list of defects for the different irradiation types helps to foresee the detector performance under high fluences.

A new line of material characterization investigating nitrogen-enriched high resistivity silicon wafers with the aim to understand the impact of nitrogen on the defect formation was created. The nitrogen-enriched wafers show a reduced production of the concentrations of some radiations centers[5]. Efforts to fabricate NDFZ (Nitrogen Doped FZ silicon) detectors and study its performance after irradiation are ongoing.

\section{Full Detector System}

Fig. 2 shows collected charge measurements for n-in-p and p-in-n detectors irradiated at different fluences (protons and neutrons). Fig. 2 shows higher collected charge for $n$-in-p detectors than p-in-n. N-in-p sensors collect electrons, which are faster than holes, can multiply and the trapping probability for electrons decreases after annealing. FZ n-in-p microstrip sensors show no reverse annealing in charge collection measurement for neutron and proton irradiation[9]. After all those results, RD50 collaboration recommended for the HL-LHC experiments the use of n-in-p 


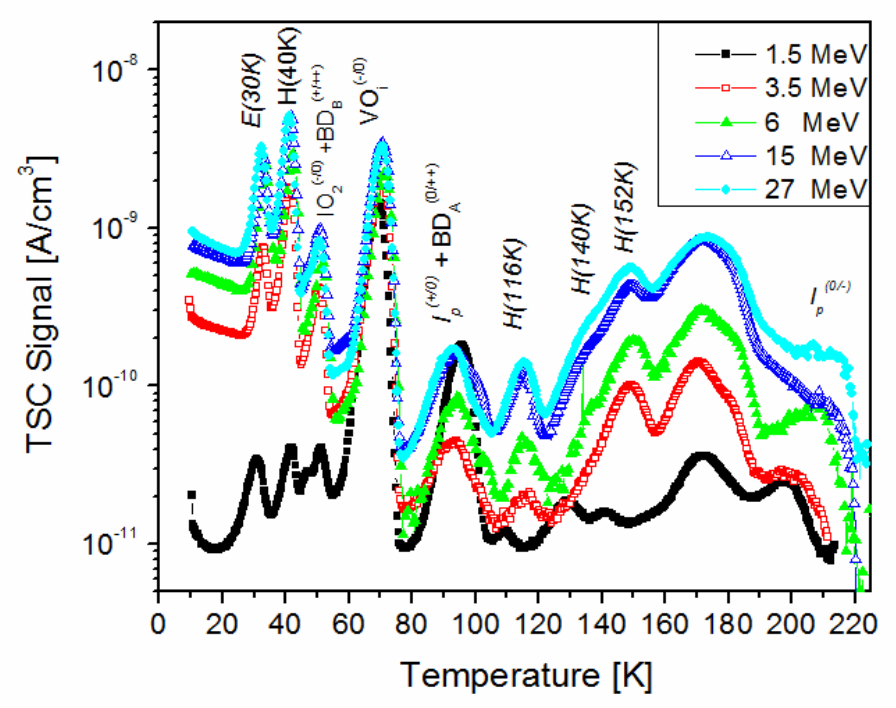

Figure 1: TSC measurement for different fluences of electron irradiation (from 1.5 MeV to 27 MeV)[4].

detectors for high radiation environments. Currently, n-in-p sensors are the baseline for ATLAS and CMS silicon strip tracking upgrades.

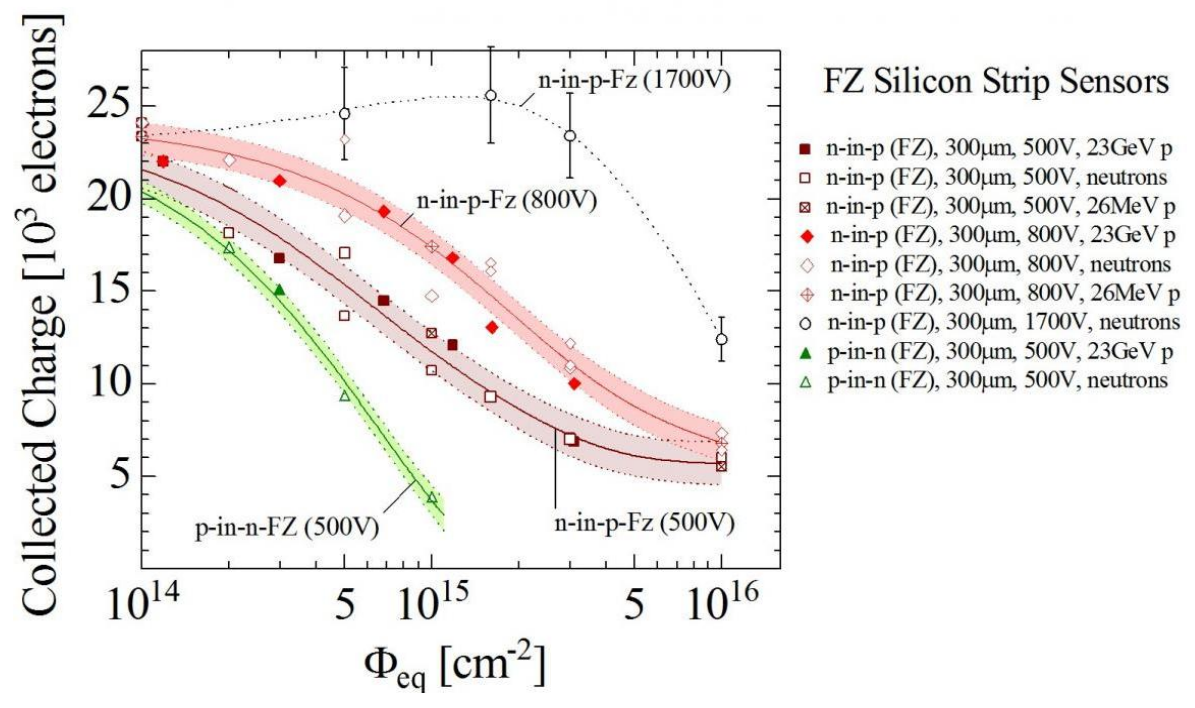

Figure 2: Collected charge for n-in-p and p-in-n radiated strip detectors $[6,7,8]$.

A further important activity is the work on the understand of charge multiplication mechanisms due to high electric fields in irradiated sensors arising from the high defect concentrations. Such effects have been observed in $[10,11,12]$ and are subject to further studies.

New requirements in radiation hardness arising from e.g. the intention to use silicon detectors in the very forward region of experiments in hadron calorimeters have asked for testing devices after extreme fluences. In Ref [13] first tests for sensors irradiated to $1 \times 10^{17} \mathrm{n}_{\mathrm{eq}} / \mathrm{cm}^{2}$ are presented that 
still show some charge collection.

\section{Different characterization techniques}

Different techniques have been developed to characterize the sensors. Transient Current Technique (TCT) shows the signal of the particle with good time resolution, allowing the reconstruction of the electric field inside the detector bulk. Edge-TCT is a TCT measurement which locates the laser at the edge of the sensors, and e-TCT gives information about the depleted volume under the detector[14]. E-TCT with the laser beam directed parallel to the strip gives a good measurement of the weighting field of the strip[15].

Two Photon Absorption (TPA)-TCT technique allows electron transitions that may be impossible to excite with one photon (but they are reachable with two photons). TPA-TCT technique uses a femto-second pulsed laser setup to increase the probability of TPA by $10^{5}[16,17]$. TRACS is a new software designed to simulate TCT measurements and it shows a good agreement with TCT and TPA measurements[18].

Weigthfield2 is a simulation software available from [19]. It simulates strip and pad geometries and calculates the drift and weighting field for detectors with and without gain[20].

Technology Computer Aided Design (TCAD) simulation toolkits assist the detector development and the good agreement with the defect irradiation helps to predict the performance of new structures, avoiding high electric field regions that might lead to an low break donw voltage. Thus the defect characterization and the consistent set of defect centers is the base for the implementation of traps for TCAD simulations[21].

\section{Development of new silicon structures}

Future upgrades for LHC demand new silicon detectors to adjust to the upcoming requirements of the electronics. In this section the new structures currently in development will be explained.

\subsection{Low Resistance Strip Sensors}

Coupling capacitors used in AC coupled strip detectors can get damaged due to the beam loss because of a large charge deposition. Low resistance strip sensors are strip detectors with an aluminum deposition on top of the implant. The metal layers are deposited on top of the implant before the coupling capacitance is defined. This technique allows a drastic reduction of their resistance avoiding damage in case of beam lost[22].

\subsection{HV-CMOS}

HV-CMOS are sensors built on low resistivity p-type wafers, and they consist on a deep and wide $n$-well that partially depletes the highly doped region underneath. The charge is collected by drift within a region of 10-20 $\mu \mathrm{m}$ [23]. HV-CMOS technology is interesting since the fabrication of the detectors is low cost and uses comercial electronic processes.

E-TCT measurements for HV-CMOS show the three different regions of collected charges, the one closer to the electrode consist on the primary signal where the collection is given by drift. The 
central part has a partial collection dominated by drift and diffusion, whereas most of the charge created in the bottom of the detector is recombined[24].

Unirradiated and radiated HV-CMOS sensors have been tested among groups of the collaboration. Fig. 3 shows e-TCT measurements of HV-CMOS unirradiated and irradiated at different bias voltages.
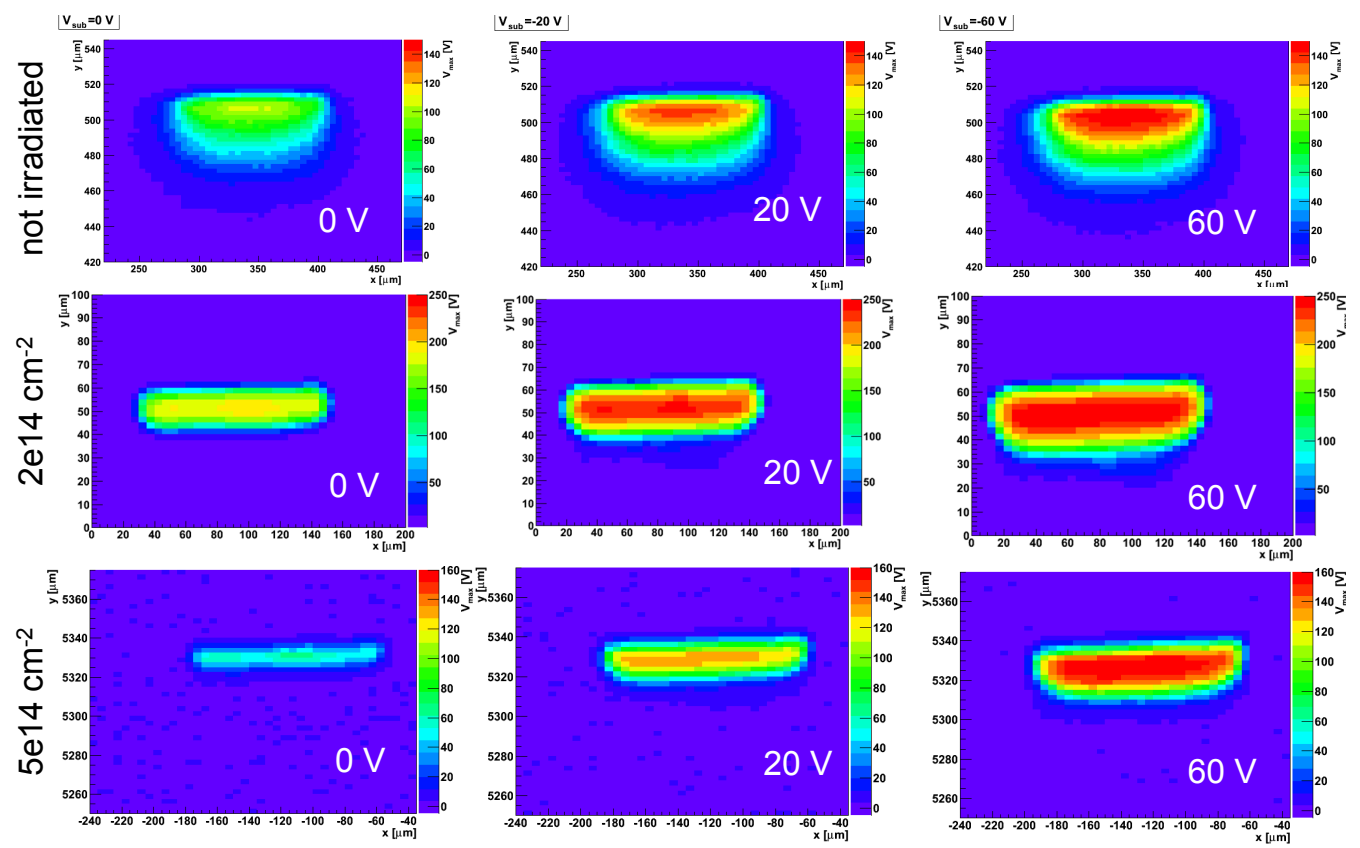

Figure 3: E-TCT scans for HV-CMOS sensors at different bias voltages for unirradiated devices and detectors irradiated at fluences $2 \times 10^{14} \mathrm{~cm}^{-2}$ and fluences $5 \times 10^{14} \mathrm{~cm}^{-2}[25]$.

\subsection{D sensors}

3D detectors were first used in a LHC experiment for the IBL in the ATLAS upgrade[26]. The electronics requirements for the LHC upgrades demand smaller pixel detectors and smaller column diameters, which challenges the 3D technology used for the IBL[27]. The smaller pixel size will decrease the distance between columns as well as the full depletion voltage of the 3D detectors. Fig. 4 shows a TCAD simulation for the new ATLAS upgrade pixel size for irradiated detectors up to fluences of $2 \times 10^{16} \mathrm{n}_{\mathrm{eq}} / \mathrm{cm}^{2}$. The radiation trap models used for that simulation are reported in [29]. The simulated particle is a MIP that impinge the detector parallel to the columns located between the $\mathrm{n}$ and $\mathrm{p}$ columns. The collected charge at fluences of $2 \times 10^{16} \mathrm{n}_{\mathrm{eq}} / \mathrm{cm}^{2}$ predicted by the simulations is up to $30 \%$ of the total charge.

The new demanding geometries of the 3D detectors lead to new challenging fabrication techniques. CNM-Barcelona is working on a Cryogenic DRIE that should increase the aspect ratio of the columns[30]. Thinner 3D detectors are planned to be fabricated on SOI wafers[31].

\subsection{LGAD}

Low Gain Avalanche Detectors (LGAD)[32] are detectors fabricated with internal gain as Avalanche Photo Diodes. They are fabricated as a n-in-p detector with a multiplication layer (a 
50umx50umx200um

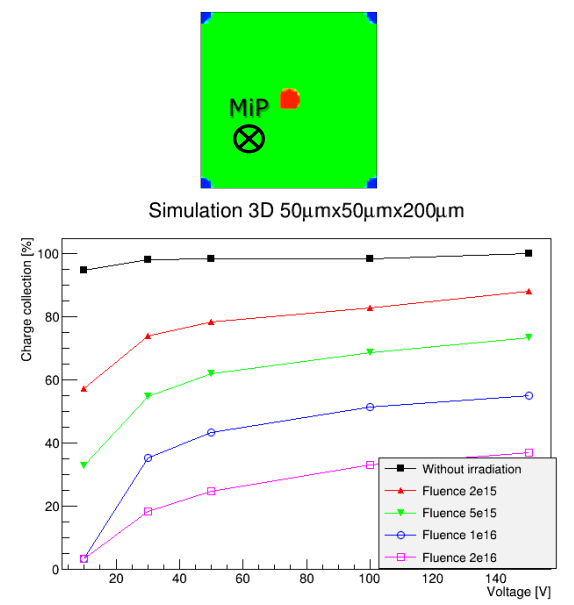

100umx25umx200um
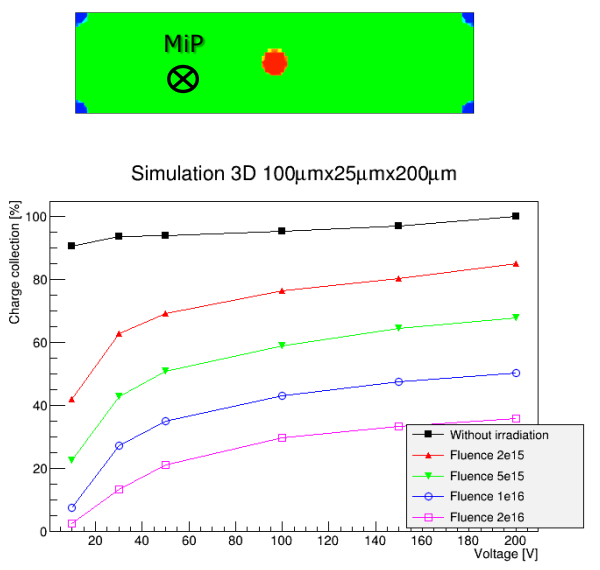

Figure 4: Simulations with Synopsys Sentaurus TCAD for the ATLAS 3D detectors[28]. The simulations show the collected charge for a MIP crossing between the two columns for different fluences.

boron implant) that brings a localized high electric field region that leads to a multiplication mechanism. Fig. 5 shows a sketch of the LGAD fabricated sensors.

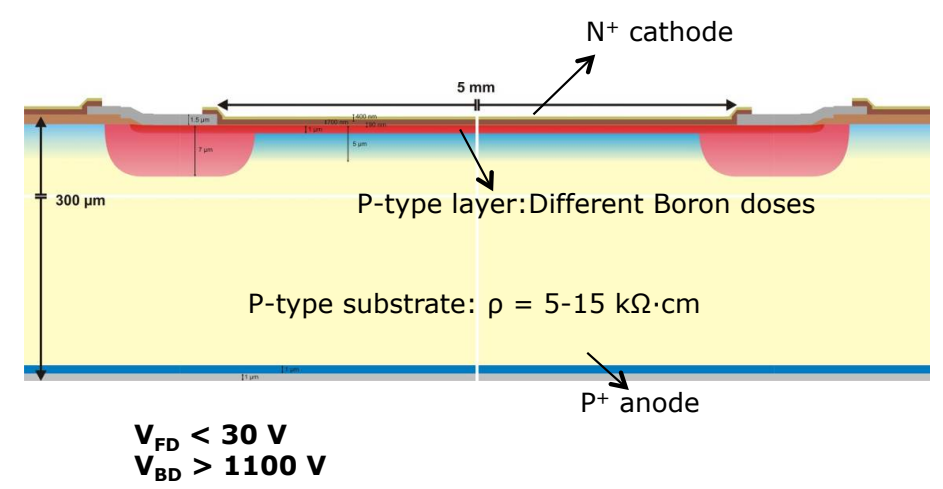

Figure 5: Sketch of the LGAD pads. n-type doped silicon is in red and p-type doped silicon is in blue.

Those detectors have reported internal gain up to 20 times the signal of a n-in-p detector (fig. 6 shows the charge versus voltage of an LGAD). Fig. 7 shows TCT measurements for LGAD compared to standard PIN detectors with pulses induced by a radioactive source $\left({ }^{241} \mathrm{Am}\right)$. The first pulse corresponds to the collected electrons and the slower and larger pulse (after $4 \mathrm{~ns}$ ) for LGAD detectors corresponds to the holes. The LGAD samples had different boron implant profiles that lead to different gains, hence, the difference in height between the signals.

Ref. [33] shows that the gain decreases with irradiation. This effect is related to a loss of the multiplication layer (the boron beneath the contact) due to irradiation, and new attempts of LGAD fabricated with gallium instead of boron are ongoing.

A possible application for LGAD detectors is timing, since thinner detectors provide faster signal and precise location information (called Ultra Fast Silicon Detectors UFSD[36]). But thin- 


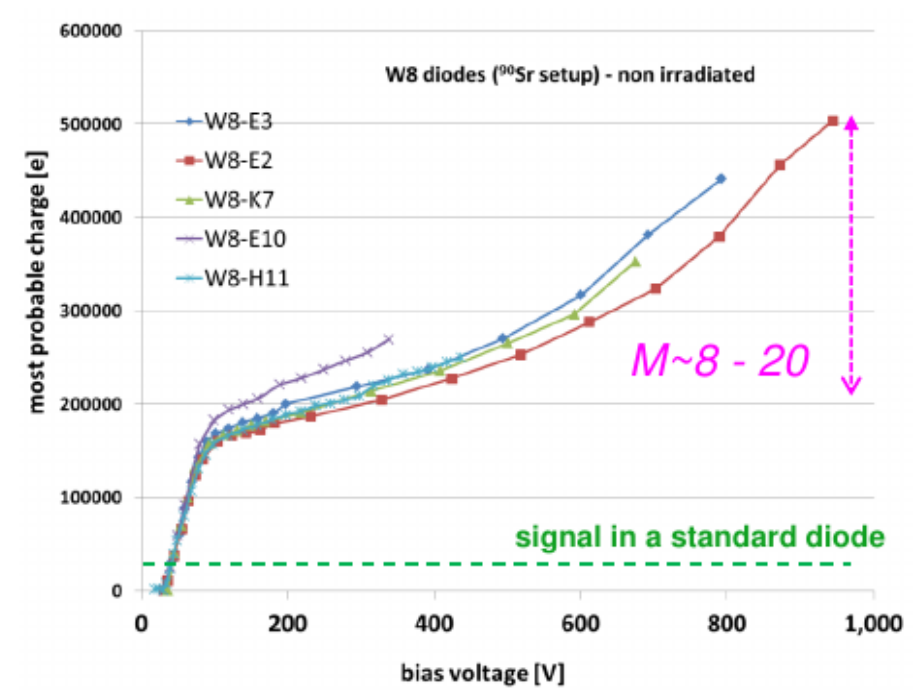

Figure 6: Gain measurements for LGAD diodes with ${ }^{90} \mathrm{Sr}$ radioactive source for different voltages[34].

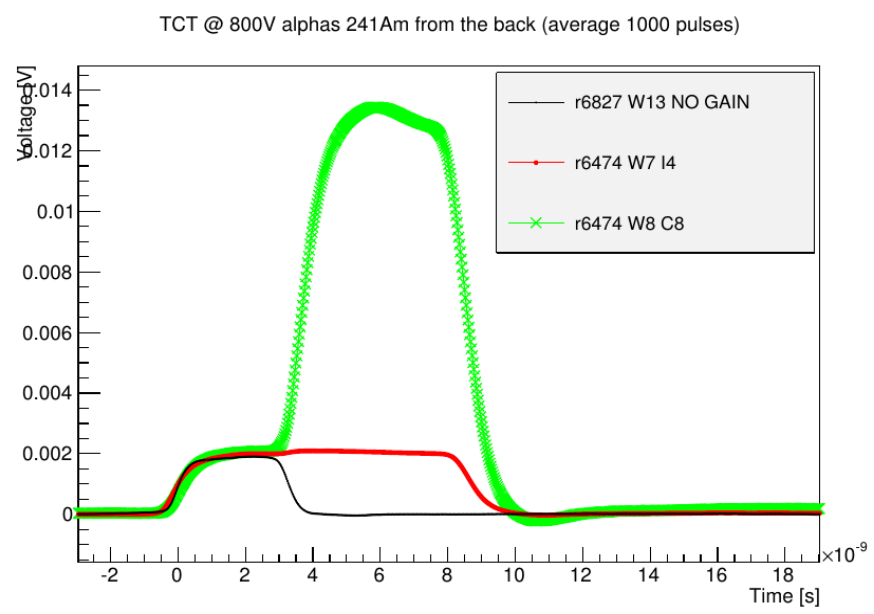

Figure 7: TCT measurement of three diodes. The diode corresponding to the black line has no intrinsic gain and the other two are LGAD with different boron concentrations. The initial pulse corresponds to the signal without gain, and it is equal for the three detectors. The slower pulse (from $4 \mathrm{~ns}$ to $10 \mathrm{~ns}$ ) corresponds to the multiplied signal for LGAD[35].

ner wafers provide smaller signals, thus a project among RD50 collaboration groups consisted on LGAD detectors fabricated on thin epitaxial wafers. Results among groups of the collaboration showed low gain measurements[37, 38]. New efforts to fabricate segmented LGAD detectors are ongoing, such as fabricating the multiplication layer on the backplane as in p-in-p detector, hence, the multiplication layer will be homogeneous for all the segmented area. Those p-in-p detectors are called iLGAD (inverted LGAD)[39] and they will have to be fabricated with a two sided process. Since p-in-p detectors would deplete from the backside, after irradiation the depleted volume would decrease, not reaching the segmented electrodes. Furthermore, p-in-p detectors collect holes that are slower and have higher trapping probability, thus they are not ideal as radiation hard detectors. 
However, thin iLGAD are studied for timing capabilities.

\section{Summary}

The RD50 collaboration works to develop radiation hard silicon detectors for high energy applications. During the upcoming upgrades of the LHC, the inner tracker detectors will receive fluences up to $2 \times 10^{16} \mathrm{n}_{\mathrm{eq}} / \mathrm{cm}^{2}$. The collaboration exchanges knowledge and organizes test beams, sensor projects and irradiation campaigns.

The collaboration explores different fields, linking the microscopic defects (building a consistent list) with the macroscopic measurements. The collaboration groups have developed different characterization techniques such as TCT, E-TCT, TPA-TCT or parallel strip measurement. Efforts in investigating the charge multiplication effects are made to allow its exploitation. New structures studied are slim and active edges, 3D-detectors, HV-CMOS, LGAD, and low-R strip sensors.

\section{Acknowledgments}

This research has been partially financed by Spanish Ministry of Economy and Competitiveness through grant FPA2013-48308-C2-2-P. The author would like to thank the collegues of the RD50 collaboration for the material and support.

\section{References}

[1] CERN R\&D proposal, LHCC 2002-003/P6, 15.2.2002, http://www.cern.ch/rd50

[2] R. Radu et al., Radiation Damage in n-type silicon diodes after electron irradiation with energies between 1.5 MeV and 15 MeV, NIMA 730 (2013) 84-90.

[3] I. Pintilie et al., Cluster related hole traps with enhanced-field-emission-the source for long term annealing in hadron irradiated Si diodes, Appl. Phys. Lett. 92, 024101, 2008.

[4] R. Radu et al., Investigation of point and extended defects in electron irradiated silicon-Dependence on the particle energy, J. Appl. Physics 117, 164503, 2015.

[5] P. Kamiński et al., First results on properties and concentrations of radiation defect centers in nitrogen-enriched, high-resistivity silicon, 25th RD50 workshop, CERN, November 2014.

[6] M. Moll, Recent advances in the development of radiation tolerant silicon detectors for the Super-LHC In Como 2009, Astroparticle, Particle and Space Physics, Detectors and Medical Physics Applications 101-110, October 2009.

[7] G. Casse, CCE studies in silicon, Vertex Proceedings of science, July 2008.

[8] I. Mandić et al., Measurement of anomalously high charge collection efficiency in $n+p$ strip detectors irradiated by up to $1 \times 10^{16} \mathrm{n}_{\mathrm{eq}} / \mathrm{cm}^{2}$, NIMA 603 (2009) 263-267.

[9] G. Casse et al., Effects of accelerated annealing on p-type silicon micro-strip detectors after very high doses of proton irradiation, NIMA 568 (2006) 46-50.

[10] G. Casse et al., Enhanced efficiency of segmented silicon detectors of different thicknesses after proton irradiations up to $1 \times 10^{16} \mathrm{n}_{\mathrm{eq}} / \mathrm{cm}^{2}$, NIMA 624 (2010) 401-404. 
[11] I. Mandić et al, Observation of full charge collection efficiency in heavily irradiated $n+p$ strip detectors irradiated up to $3 \times 10^{15} \mathrm{n}_{\mathrm{eq}} / \mathrm{cm}^{2}$, NIMA 612 (2010) 474-477.

[12] M. Kohler et al., Comparative measurements of highly irradiated n-in-p and p-in-n 3D silicon strip detectors, NIMA 659 (2011) 272-281.

[13] M. Mikuž et al., Electric field and mobility in extremely irradiated silicon, 25th RD50 workshop, CERN, November 2014.

[14] G. Kramberger et al., Investigation of Irradiated Silicon Detectors by Edge-TCT, IEEE Transactions on Nuclear Science, 57, 4, August 2010.

[15] I. Mandić et al., E-TCT measurement with laser beam directed parallel to strips, 25th RD50 workshop, CERN, November 2014.

[16] R. Palomo et al., Two Photon Absorption \& Carrier Generation in Semiconductors, 25th RD50 workshop, CERN, November 2014.

[17] I. Vila et al., A novel Transient-Current-Technique based on the Two Photon Absorption process, 25th RD50 workshop, CERN, November 2014.

[18] P. de Castro, TRACS: Transient Current Simulator, 25th RD50 workshop, CERN, November 2014.

[19] http://cern.ch/weightfield2.

[20] N. Cartiglia, Ultra-Fast Silicon Detector, 25th RD50 workshop, CERN, November 2014.

[21] T. Peltola, RD50: Simulation of radiation-induced defects, Proceedings in this conference.

[22] M. Ullan et al., Low-Resistance Strip Sensors for Beam-Loss Event Protection, NIMA 765 (2014) 252-257.

[23] I. Perić, Habilitation thesis, Ruprecht -Karls -University of Heidelberg, April 2010.

[24] M. Fernandez Garcia, Edge-TCT characterization of irradiated HV-CMOSv3 sensors, 25th RD50 Workshop, CERN, November 2014.

[25] G. Kramberger, Edge-TCT studies of irradiated HVCMOS sensor (an update), 25th RD50 workshop, CERN, November 2014

[26] J. Lange, Recent Progress on 3D Silicon Detectors, Proceedings in this conference.

[27] G. Pellegrini et al., 3D double sided detector fabrication at IMB-CNM, NIMA 699 (2013) 27-30.

[28] M. Baselga et al., New 3D fabrication at CNM for the ATLAS Pixel upgrade, 10th Trento Workshop, February 2015.

[29] D. Pennicard, 3D detectors for Synchrotron applications, PhD thesis, Glasgow 2010.

[30] B. Wu et al., High aspect ratio silicon etch: A review, J. Appl. Physics, 108, 051101 (2010).

[31] F. Garcia, Ultra Thin 3D Silicon Detector for Plasma Diagnostics at the ITER Tokamak, Nuclear Science Symposium and Medical Imaging Conference (NSS/MIC), 2011 IEEE, 199-201.

[32] G. Pellegrini et al., Technology developments and first measurements of Low Gain Avalanche Detectors (LGAD) for high energy physics applications, NIMA 765 (2014) 12-16.

[33] G. Kramberger et al., Radiation effects in Low Gain Avalanche Detectors after hadron irradiations, JINST 10, July 2015, 07006.

[34] G. Kramberger et al., Radiation hardness of Low Gain Amplification Detectors (LGAD), 24th RD50 workshop, Bucharest, June 2014. 
[35] H. Sadrozinski et al., Sensors for ultra-fast silicon detectors, NIMA 765 (2014) 7-11.

[36] A. Seiden, Ultra-fast silicon detectors, Proceedings in this conference.

[37] E. Cavallaro et al., Measurements of LGAD segmented devices, 25th RD50 workshop, CERN, November 2014.

[38] M. Baselga et al., Measurements with strips LGAD p-type detectors fabricated at CNM-Barcelona, 10th Trento Workshop, February 2015.

[39] S. Hidalgo et al., New iLGAD detector development at CNM, 26th RD50 workshop, Santander, June 2015. 